\title{
Effects of genetic deletion of the Kv4.2 voltage- gated potassium channel on murine anxiety-, fear- and stress-related behaviors
}

\author{
Carly Kiselycznyk ${ }^{1 *}$, Dax A Hoffman² and Andrew Holmes ${ }^{1}$
}

\begin{abstract}
Background: Potassium channels have been proposed to play a role in mechanisms of neural plasticity, and the Kv4.2 subunit has been implicated in the regulation of action-potential back-propagation to the dendrites. Alterations in mechanisms of plasticity have been further proposed to underlie various psychiatric disorders, but the role of Kv4.2 in anxiety or depression is not well understood.

Methods: In this paper, we analyzed the phenotype Kv4.2 knockout mice based on their neurological function, on a battery of behaviors including those related to anxiety and depression, and on plasticity-related learning tasks.

Results: We found a novelty-induced hyperactive phenotype in knockout mice, and these mice also displayed increased reactivity to novel stimulus such as an auditory tone. No clear anxiety- or depression-related phenotype was observed, nor any alterations in learning/plasticity-based paradigms.

Conclusions: We did not find clear evidence for an involvement of Kv4.2 in neuropsychiatric or plasticity-related phenotypes, but there was support for a role in Kv4.2 in dampening excitatory responses to novel stimuli.
\end{abstract}

\section{Background}

The excitability and functional plasticity of specific brain circuits in cortical, limbic, and midbrain regions is thought to mediate behavioral responses to environmental threats, and to enable adaptation to stressors. At the cellular level, voltage- and calcium-activated potassium $(\mathrm{K}+)$ channels provide an important means of modulating neuronal activity and synaptic plasticity. Their function is further refined via the presence of multiple $\mathrm{Kv}$ primary and auxiliary subunits that differ in their voltage-dependence, post-translational regulation, sub-cellular localization and regional pattern of expression in the brain to accomplish distinct physiological functions.

A-type $\mathrm{K}+$ channels composed of Kv4 subunits transmit a rapidly activating and inactivating current [1]. Kv4.2 mRNA is expressed in the periphery and in various brain regions involved in mediating stress-related behaviors, including the medial prefrontal cortex (mPFC), hippocampus and hypothalamus [2]. In CA1

\footnotetext{
* Correspondence: kiselycznykc@mail.nih.gov

'Laboratory of Behavioral and Genomic Neuroscience, National Institute on Alcohol Abuse and Alcoholism, NIH, Bethesda, MD, USA

Full list of author information is available at the end of the article
}

hippocampal neurons, Kv4.2 protein is primarily localized on dendrites [3] where they exert their strongest functional effects [4]. Gene deletion of Kv4.2 (Kcnd2) in mice eliminates most of the A-type $\mathrm{K}+$ current in hippocampal and cortical neurons [5-7], and increases back-propagation of the action potential from the axon to the somatodendritic region [6]. These and other effects of Kv4.2 knockout (KO) may be countered to some extent by compensatory increases in the expression of other $\mathrm{Kv}$ subunits [8], and increased inhibitory transmission in the hippocampus [9]. Notwithstanding, loss of $\mathrm{Kv} 4.2$ function produces a net increase in neuronal, and particularly dendritic, hyperexcitability in the mPFC and hippocampus, and possibly other brain regions in which this subunit is expressed.

Synaptic plasticity is also altered by Kv4.2 deletion, as evidenced by the enhanced CA1 hippocampal long-term potentiation (LTP) and increased threshold for longterm depression (LTD) seen in Kv4.2 (KO) mice [6,10]. Further demonstrating an important contribution to hippocampal plasticity, Kv4.2 channels are internalized during LTP induction, and Kv4.2 overexpression or deletion alters synaptic expression of N-Methyl-D-

\section{() Biomed Central}


aspartate (NMDA) receptor subunits (GluN2A, GluN2B) to respectively constrain or promote hippocampal LTP $[11,12]$. Conversely, NMDAR throughput decreases hippocampal Kv4.2 through degradation $[13,14]$ whereas concurrently causing an increase in Kv4.2 translation [15]. The reciprocal relationship between Kv4.2 and NMDA receptor subunits is particularly intriguing in the context of prior studies showing that gene $\mathrm{KO}$ of GluN2A [16] or GluN2B [17] disrupts anxiety-related behaviors and adaptations to stress.

Behaviorally, Kv4.2 KO mice are viable and appear grossly normal [18]. However, Kv4.2 KO mice show augmented nociceptive responses to thermal and mechanical stimulation [8] and increased sensitivity to the pro-convulsive effects of kainate, with no apparent increase in spontaneous seizures [19]. Furthermore, in a recent study assessing Kv4.2 KO mice on a $129 \mathrm{~S} 6 / \mathrm{SvEv}-$ Tac (129S6) background on a range of behavioral assays, Lockridge et al. found that the $\mathrm{KO}$ mice had reduced grip strength on a wire-hang test and increased locomotor activity in a brightly illuminated open field, but not in a dimly illuminated one [20]. Although the KO mice displayed an increased tendency to enter into the aversive central area of the open field, consistent with an anxiolytic-like phenotype, the mutants were no different from the wild-type (WT) controls in the increased plusmaze test for anxiety-such as behavior. This study also found that Kv4.2 KO mice showed increased immobility in the forced-swim test (FST) (but not the tail-suspension test) for 'depression-related' behavior, and were insensitive to the anti-immobility effects of the antidepressant fluoxetine, but not imipramine or desipramine, in the FST. In addition, ex vivo slice physiological recordings indicated that a form of 5-hydroxytryptamine (5-HT)2A receptor-mediated excitatory synaptic transmission in mPFC pyramidal neurons was attenuated in Kv4.2 KO mice after a single forced swim exposure, but only after repeated exposures in WT controls. Based on these findings, Lockridge et al. concluded that Kv4.2 deletion produces abnormal behavioral responses to stress, and suggested that this might be related to excessive 5 -HT release.

The aim of the present study was to confirm and extend the characterization of $\mathrm{Kv} 4.2 \mathrm{KO}$ mice in stressand other 'emotion'-related phenotypes. $\mathrm{KO}$ mice and WT littermate controls were compared for a battery of sensory and neurological tests (including hot-plate nociception, acoustic startle and prepulse inhibition of startle, and home-cage locomotion), exploratory and anxiety-related behaviors (novel open field, light/dark exploration, elevated plus-maze tests), pavlovian fear conditioning and extinction, and 'depression-related' behavioral responses (single and repeated inescapable forced-swim exposure). Phenotyping was conducted after the mutants were backcrossed onto a C57BL/6J background strain. This is important because genetic background can strongly influence the phenotype of mutant mice [21,22], and the aforementioned studies by Lockridge and colleagues tested the $\mathrm{KO}$ mice on a different genetic background (129S6).

\section{Methods}

Experimental procedures were performed in accordance with the NIH Guide for Care and Use of Laboratory Animals and were approved by the local Animal Care and Use Committee.

\section{Animals}

Kv4.2 KO mice were generated in a 129 S6 genetic background as described previously [19]. For the current experiments, mice were backcrossed to the C57BL/6J strain for nine generations. To control for potential effects of maternal genotype [22], KO mice and WT controls were littermates bred from heterozygous parents. Males and females were used. Mice were housed in same-sex groupings in a temperature- and humiditycontrolled vivarium under a 12-hour light/dark cycle (lights on at 06.00 hours) and testing conducted during the light phase after 1 hour of acclimation to the test room. Three separate, naïve cohorts were tested. One cohort of mice was screened on a battery of neurological measures, including prepulse inhibition and home-cage activity, and basal home-cage corticosterone levels. A second cohort was tested on a battery of assays for anxiety-, fear- and stress-related phenotypes, with putatively more stressful tests conducted later in the sequence and at least 6 days between each test: novel open field, light/ dark exploration, elevated plus-maze, pavlovian fear conditioning and extinction, and forced-swim test/swiminduced corticosterone response. A third cohort was tested for behavioral responses to repeated inescapable forced swims. The experimenter remained blinded to genotype during testing. The number of mice tested in each assay is given in the corresponding figure legends.

\section{Initial phenotypic screen \\ Neurological test battery}

Functional observational battery Gross physical and neurological abnormalities were examined using a simple functional observation battery, as described previously $[16,23,24]$. Basic physical health was evaluated by examining for missing whiskers, bald patches, piloerection, exophthalmus, straub tail, kinked tail, kyphosis, lordosis, core body temperature, reactivity to handling, and body weight. Simple sensory reflexes were measured via orienting responses to an approaching hand and to physical touch via palpebral closure on touch of the eye, twitch of pinna on touch, and an orienting response to 
tail pinch. Mice were observed for splayed limbs, and forepaw and hind limb clutch when suspended upsidedown by the tail, and wild running, freezing, trembling, sniffing, licking, rearing, jumping, seizures, defecation, urination, head bobbing, circling, abnormal gait, retropulsion, and prancing forelimbs when placed in a novel, bare, standard holding cage $(130 \times 135 \times 350 \mathrm{~mm})$ for 1 minute.

Nociception Pain perception was tested using the hotplate test. a flat plate (Columbus Instruments, Columbus, $\mathrm{OH}, \mathrm{USA}$ ) was heated to $55^{\circ} \mathrm{C}$, and the mouse placed upon it. The latency to first hind-paw lick was manually timed, with a maximum response latency of 30 seconds. and genotypes statistically compared via Student's t-test.

Acoustic startle and prepulse inhibition of startle Acoustic startle and prepulse inhibition of startle (PPI) was tested as described previously [25]. The mouse was placed in a clear Plexiglas holding cylinder (San Diego Instruments SR-LAB System; San Diego, CA, USA) for an acclimation period of 5 minutes. A $65-\mathrm{dB}$ broadband background noise was presented during acclimation and throughout the test session. Five pulse-alone trials (120 $\mathrm{dB}$ broadband sound pulse for $40 \mathrm{~ms}$ ) began and ended the session, and were not included in the analysis. Testing consisted of presentation of $120 \mathrm{~dB}$ startle trials and prepulse + startle trials (noise prepulse sound for $20 \mathrm{~ms}$, followed $100 \mathrm{~ms}$ later by the $40 \mathrm{~ms} 120-\mathrm{dB}$ broadband sound pulse). There were three different prepulse intensities (3, 6 and $12 \mathrm{~dB}$ above background), each presented 10 times with a variable interval (range 12-30 seconds) between each presentation. There were vie pulse-alone trials before and after each prepulse + startle trial. Basal activity in the startle chambers was measured during the no-stimulus trials. Startle amplitude was measured starting from the onset of the startle stimulus via wholebody vibrations (sampled every $1 \mathrm{~ms}$ over $65 \mathrm{~ms}$ ), transduced into analog signals by a piezoelectric unit attached to the platform on which the cylinders rested. This was used to derive an average startle amplitude over the $65 \mathrm{~ms}$ recording period. PPI at each prepulse intensity was calculated as:

$$
100-\frac{\text { startle response for prepulse }+ \text { startle trials }}{\text { startle response for startle }- \text { alone trials }} \times 100
$$

The effects of genotype and PPI intensity were analyzed using two-way analysis of variance (ANOVA), with repeated measures for PPI intensity.

Home-cage activity The mouse was individually housed in a standard holding cage as before, and left undisturbed for a 48-hour acclimation period under normal vivarium conditions. Activity was measured over 24 hours using a photocell-based activity monitor (Opto
M3; Columbus Instruments, Columbus, OH, USA) and expressed as the 12-hour average activity during the light and dark phase, as described previously [26]. The effects of genotype and circadian phase were analyzed using two-way ANOVA, with repeated measures for cycle.

\section{Battery of anxiety-, fear-, and stress-related behaviors Anxiety-related behaviors}

Novel open field The novel open-field test [27] was conducted as described previously [28] in a $400 \times 400 \times 350$ mm square arena (60 lux) constructed of white Plexiglas. Testing was conducted under $65 \mathrm{~dB}$ white noise to minimize external noise disturbances (Sound Screen, Marpac Corporation, Rocky Point, NC, USA). The mouse was placed in the perimeter and allowed to explore the apparatus for 30 minutes. Total distance traveled and time spent in the center square $(200 \times 200 \mathrm{~mm})$ was measured by a video-tracking system (Ethovision; Noldus Information Technology Inc., Leesburg, VA, USA).

Light-dark exploration test The light-dark exploration test [24] was conducted as described previously [29]. The mouse began the test in an opaque black Plexiglas shelter $(390 \times 130 \times 160 \mathrm{~mm})$ with a $130 \times 80 \mathrm{~mm}$ opening at floor level that opened onto a large white Plexiglas square arena $(390 \times 390 \times 350 \mathrm{~mm})$ illuminated to approximately 90 lux. Percentage time spent in the lighted compartment, frequency of entering the lighted compartment, and total distance traveled in the entire apparatus over a 15 min session were measured by a video-tracking system (Ethovision Noldus Information Technology Inc). Mice that did not enter the lighted compartment were recorded as having a latency to exit time of 900 seconds. Genotypes were compared using Student's t-test.

Elevated plus-maze The elevated plus-maze test [30] was conducted as described previously [31]. The apparatus consisted of two open arms $(300 \times 50 \mathrm{~mm}$; $90 \mathrm{lux})$ and two closed arms $(300 \times 50 \times 150 \mathrm{~mm} ; 20 \mathrm{lux})$, extending from a $50 \times 50 \mathrm{~mm}$ central area and elevated $200 \mathrm{~mm}$ from the ground (San Diego Instruments, San Diego, CA, USA). The walls were made from black acrylonitrile butadiene styrene (ABS) plastic and the floor from white ABS plastic. A $50 \mathrm{~mm}$ high raised lip around the perimeter of the open arms prevented the mice from falling off the maze. Testing was conducted under $65 \mathrm{~dB}$ white noise (Sound Screen, Marpac Corporation, Rocky Point, NC, USA) to minimize external noise disturbance. The mouse was placed in the center of the maze facing an open arm, and allowed to explore the apparatus for 6 minutes. Time spent in the open arms and number of entries into the open and closed arms were measured by a video-tracking system (Ethovision Noldus Information 
Technology Inc). Genotypes were compared using Student's t-test.

\section{Fear-related behaviors}

Pavlovian fear conditioning and extinction Pavlovian fear conditioning and extinction was assessed as described previously $[32,33]$. The mouse was placed in context A: a $270 \times 270 \times 110 \mathrm{~mm}$ chamber with transparent walls and a metal rod floor. To provide a distinctive olfactory environment, the chamber was cleaned between subjects with a $79 \%$ ethanol/20\% water $/ 1 \%$ vanilla extract solution. After acclimation period of 180 seconds, the mouse received three pairings (interval of 60-120 seconds after each pairing) of an auditory tone (30 seconds, $80 \mathrm{~dB}$, white noise) and footshock (2 seconds, $0.6 \mathrm{~mA}$ scrambled footshock), in which the shock was presented during the last 2 seconds of the tone. The presentation of stimuli was controlled by a freeze monitoring system (Med Associates Inc, Georgia, VT, USA).

Twenty-four hours later, expression of fear to the tone and subsequent within-session extinction was tested. Mice were placed in context B: a novel context (Plexiglas cylinder with black/white-checkered walls and a solid floor, cleaned with a $1 \%$ acetic acid/99\% water solution) housed in a novel room. After an initial acclimation period of 180 seconds, the mouse received 500 presentations of the tone alone (each tone for 30 seconds, with a no-stimulus interval of 5 seconds). Twenty-four hours later, extinction retrieval was probed with three tone presentations in context A. Four hours later, mice were presented with three tones in context B to assess fear renewal.

Freezing (no visible movement except that required for respiration) in response to the tone was manually scored every 5 seconds and converted to a percentage:

(number of freezing observations/total number of observations $\times 100$ )

Freezing during extinction trials were averaged into 100 five-trial blocks for analysis, and the first and last trial blocks compared. The effect of genotype and trial/ trial block during conditioning and extinction were analyzed using two-factor ANOVA, with repeated measures for trial/trial block, followed by Bonferroni post hoc tests. The effect of genotype on extinction retrieval and fear renewal were analyzed using Student's t-test.

\section{Responses to swim stressors}

\section{Forced-swim test behavior and corticosterone response}

The forced-swim test [34] was conducted as described previously [35]. The mouse was gently lowered into a $200 \mathrm{~mm}$-diameter cylinder filled with water $24 \pm 1.0^{\circ} \mathrm{C}$ for test lasting 6 minutes. Immobility (cessation of limb movements except for minor movement necessary to keep the mouse afloat) was manually scored every $5 \mathrm{sec}$ onds during minute 3 to 6 . Genotypes were compared using Student's t-test.
Mice were returned to the home cage for 30 minutes after forced swim, and then killed via cervical dislocation and decapitated. The trunk blood was collected, left to coagulate at room temperature for 1-2 hours, and then separated by centrifugation at $4^{\circ} \mathrm{C}$ for 30 seconds at $13,000 \mathrm{rpm}(20000 \mathrm{x} \mathrm{g})$. Serum was collected and analyzed for corticosterone (bound and free) using a commercial radioimmunoassay (MP Biomedicals, Orangeburg, NY) as described previously [36]. Genotypes were compared using Student's t-test.

Repeated inescapable forced swim The repeated inescapable forced-swim (riFS) test was conducted as described previously [17]. The mouse was gently lowered into a cylinder filled with water at $24 \pm 1.0^{\circ} \mathrm{C}$. The cylinder was of a larger diameter $(300 \mathrm{~mm})$ than that used for the forced-swim test used above $(200 \mathrm{~mm})$ and had a $40 \times 40 \mathrm{~mm}$ escape hole located $40 \mathrm{~mm}$ above the waterline and out of reach for the mouse. A platform (plastic wiffle ball) was magnetically held $100 \mathrm{~mm}$ below the water, also out of reach. After 1 minute, the platform was remotely released (by turning off the magnet) and quickly (within $<2$ seconds) floated vertically upwards along a pole to sit on top of the water, directly under the escape hole. If the mouse attempted to climb onto or hold the platform, it sank below the water. The test terminated 20 seconds after the platform was presented, regardless of the number of attempts by the mouse to use it to reach the escape hole. There was one trial per day (10.00 to 12.00 hours) for 10 consecutive days. Immobility was manually scored every 5 seconds during the 1 minute before platform presentation. The effect of genotype and trial were analyzed using two-way ANOVA, with repeated measures for trial.

\section{Statistical analysis}

Knockout and wildtype mice were compared using Student's t-test with the exception of the prepulse inhibition of startle, home cage activity, fear conditioning and extinction, and riFS testing, which was analyzed using a 2-factor analysis of variance (ANOVA) with Bonferroni posttest. The threshold for statistical significance was $P$ $<$.05. GraphPad Prism 5 statistical software (La Jolla, CA) was used for all statistical analysis.

\section{Results}

Normal gross behavior and neurological functions

The different genotypes did not differ on most measures of physical health, gross behavior, or neurological functions (Table 1), with the exception of increased rearing seen in $\mathrm{KO}$ mice relative to WT controls when placed in an empty cage for $1 \mathrm{~min}\left(\mathrm{t}_{(16)}=2.21, P<0.05\right)$ (Figure 1A). Hot-plate nociception was not different between genotypes (Figure 1B). KO mice also showed 
Table 1 Empty-cage behaviors, physical health, sensory reflexes, and neurological functions

\begin{tabular}{|c|c|c|}
\hline Empty-cage behaviors $^{\text {a }}$ & $\mathrm{WT}^{\mathrm{b}}$ & $\mathrm{KO}^{\mathrm{c}}$ \\
\hline Freezing & 0 & 0 \\
\hline Trembling & 0 & 0 \\
\hline Sniffing & 100 & 100 \\
\hline Licking & 0 & 0 \\
\hline Rearing & 80 & 100 \\
\hline Jumping & 0 & 0 \\
\hline Seizure & 0 & 0 \\
\hline Defecation & 60 & 25 \\
\hline Urination & 0 & 0 \\
\hline Head bobbing & 0 & 0 \\
\hline Circling & 0 & 0 \\
\hline Abnormal gait & 0 & 0 \\
\hline Retropulsion & 0 & 0 \\
\hline \multicolumn{3}{|l|}{ Physical health } \\
\hline Missing whiskers & 0 & 0 \\
\hline Bald patches & 0 & 0 \\
\hline Exopthalmus & 0 & 0 \\
\hline Straub tail & 0 & 0 \\
\hline Kinked tail & 0 & 0 \\
\hline Kyphosis & 0 & 0 \\
\hline Lordosis & 0 & 0 \\
\hline Body weight, $g^{e}$ & $23.6 \pm 0.8$ & $23.9 \pm 1.3$ \\
\hline Core body temp, ${ }^{\circ} \mathrm{C}^{\mathrm{e}}$ & $36.2 \pm 0.3$ & $36.8 \pm 0.1$ \\
\hline \multicolumn{3}{|l|}{ Sensory reflexes } \\
\hline Approach response & 100 & 100 \\
\hline Touch response & 100 & 100 \\
\hline Palpebral response & 100 & 100 \\
\hline Pinna reflex & 100 & 100 \\
\hline Tail pinch response & 100 & 100 \\
\hline \multicolumn{3}{|l|}{ Motor, neurological } \\
\hline Splayed limbs & 100 & 100 \\
\hline Forepaw clutch & 0 & 0 \\
\hline Hind-paw clutch & 0 & 0 \\
\hline
\end{tabular}

${ }^{\mathrm{a}}$ Data denote the percentage of animals showing a response unless specified otherwise,

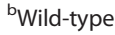

${ }^{\mathrm{c}}$ Knockout.

${ }^{\mathrm{e}}$ Mean $\pm \mathrm{SD}$

no alterations in acoustic startle compared with WT controls (Figure 1C). There was a significant effect of prepulse intensity $\left(F_{(2,32)}=35.65, P<0.01\right)$ but not of genotype, and no intensity $\times$ genotype interaction for PPI (Figure 1D). There was a significant effect of circadian phase for home-cage activity $\left(F_{(1,15)}=23.94, P<\right.$ 0.01 ), with both genotypes showing more activity during the dark phase than the light phase (Figure 1E) (after one mouse per genotype with outlying scores (> $2 \mathrm{SD}$ ) were excluded). There was no effect of genotype or phase $\times$ genotype interaction for home-cage activity.
Increased exploratory locomotion and test-specific decreased anxiety-like behavior

In the novel open-field test, time-course analysis revealed a significant effect of genotype $\left(F_{(1,18)}=13.70\right.$, $P<0.01)$ and time bin $\left(F_{(5,90)}=14.13, P<0.01\right)$, but no interaction for distance traveled (Figure 2A). KO mice traveled significantly farther than WT controls over the whole session $\left(\mathrm{t}_{(18)}=3.70, P<0.01\right)$ (Figure $2 \mathrm{~B}$ ). Genotypes did not differ in the time spent in the center of the open field (Figure 2C).

In the light/dark exploration test, genotypes did not differ in percentage time (Figure 2D) or entries into (Figure 2E) the lighted compartment during the first or last (data not shown) 5 minutes. Genotypes also did not differ in distance traveled in both compartments during the first (data not shown) or last 5 minutes of the session (Figure 2F).

In the elevated plus maze, $\mathrm{KO}$ mice spent significantly more time in the open arms $\left(\mathrm{t}_{(17)}=2.30, P<0.05\right.$ ) (Figure $2 \mathrm{G}$ ), and made significantly more entries into the open arms $\left(\mathrm{t}_{(17)}=2.52, P<0.05\right)$ (Figure $\left.2 \mathrm{H}\right)$, compared with WT controls. Genotypes did not differ in the number of entries into the closed arms (Figure 2I). One WT mouse with outlying (> $2 \mathrm{SD}$ ) was excluded from each of the three elevated plus maze measures (time in open arms, open-arm entries, closed-arm entries).

\section{Increased freezing to unconditioned auditory tones}

During conditioning, there was significant interaction between the conditioned stimulus (CS) trial and genotype $\left(F_{(1,21)}=6.28, P<0.05\right)$ for freezing to the CS tone. Post hoc tests revealed higher freezing to the first (that is, before any foot shock) and second CS trial but not to the third CS trial in KO mice (Figure 3A).

During extinction training, there was a significant effect of trial block $\left(F_{(9,189)}=15.25, P<0.01\right)$, and significant reduction in freezing to the last five tones compared with the first five $\left(F_{(1,21)}=61.35, P<0.01\right)$. However, there was no effect of genotype or trial block $x$ genotype interaction for freezing (Figure $3 \mathrm{~B}$ ).

Genotypes did not differ in freezing to the CS during either the extinction-retrieval (Figure 3C) or contextrenewal tests (Figure 3D). Freezing during the baseline pre-CS periods was negligible, and was not different between genotypes for extinction training (WT $=9.5 \pm$ $2.4, \mathrm{KO}=6.4 \pm 1.4)$ or retrieval $(\mathrm{WT}=4.2 \pm 1.2, \mathrm{KO}=$ $1.2 \pm 1.2)$.

\section{Exaggerated swim-induced corticosterone and normal 'depression-related' responses to single and repeated forced swim}

During a single forced-swim exposure, genotypes did not differ in percentage of time spent immobile (Figure 4A). However, serum corticosterone levels after this 


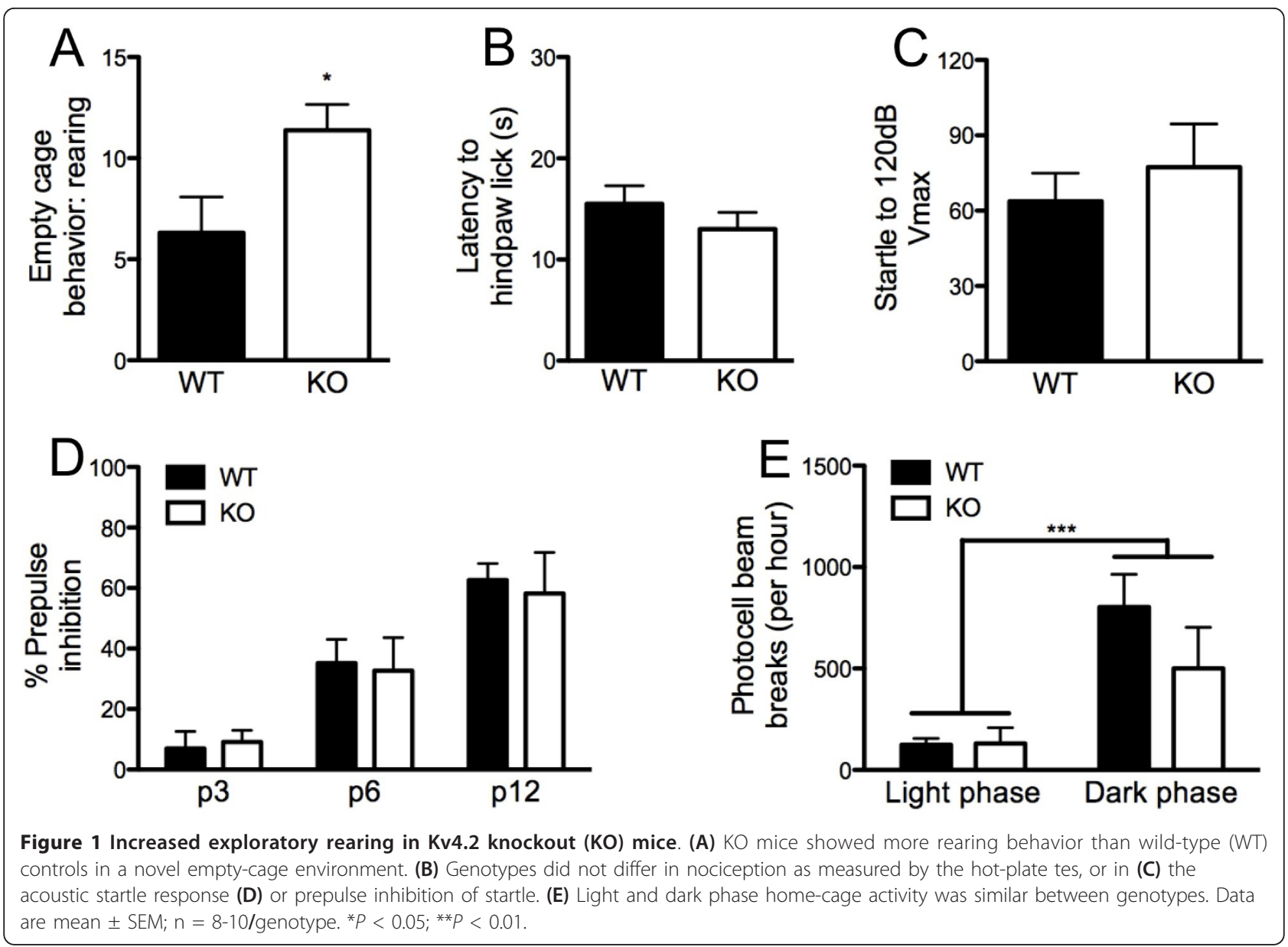

swim exposure were significantly higher in $\mathrm{KO}$ mice than WT controls $\left(\mathrm{t}_{(19)}=3.27, P<0.01\right)$ (Figure $4 \mathrm{~B}$ ). Based on this genotype difference, a separate cohort of stress-naïve mice was killed immediately on removal from the cage for corticosterone analysis. Corticosterone levels did not differ between genotypes under these non-stressed conditions (WT $=40.4 \pm 4.3 \mathrm{mg} / \mathrm{mL}, \mathrm{KO}$ $=109.5 \pm 41.8 \mathrm{mg} / \mathrm{mL}$ ). Three WT and one $\mathrm{KO}$ sample were removed because of errors in the blood-collection assay.

On the riFS test, there was a significant effect of trial $\left(F_{(9,270)}=27.85, P<0.01\right)$ but not of genotype, and no trial $\times$ genotype interaction for percentage immobility (Figure 4D).

\section{Discussion}

The aim of the current study was to examine the effects of deleting the A-type voltage-gated $\mathrm{K}+$ channel $\mathrm{Kv} 4.2$ on multiple stress- and other 'emotion'-related phenotypes. We found a complex set of significant phenotypic alterations in Kv4.2 mice. These included increased exploratory activity in novel environments, test-specific decreases in anxiety-like behavior, increased fear response to auditory stimuli, and an exaggerated corticosterone response to stress.

Consistent with previous studies [19], an initial observational battery showed no gross alterations in various measures of health or sensory function in the Kv4.2 knockout; however, this analysis did reveal increased rearing in the $\mathrm{KO}$ mice, suggestive of heightened levels of exploratory behavior. Similarly, exploratory locomotion in a novel open field was robustly increased in the $\mathrm{KO}$ mice, at least over a 30 minute session (longer sessions were not examined). These genotype effects did not seem to reflect generalized locomotor hyperactivity in the mutants, as evidenced by normal levels of activity in the familiar and low-stress environment of the home cage. Rather, these data suggest that Kv4.2 deletion produced an augmented behavioral reaction to novel and/ or stressful environments.

This behavioral phenotype may be related to the neural abnormalities that have been reported in Kv4.2 $\mathrm{KO}$ mice. For example, these mice have been found to exhibit neuronal and dendritic hyperexcitability due to increased axonal back-propagation [6]. Interestingly in this context, we have previously identified a similar 


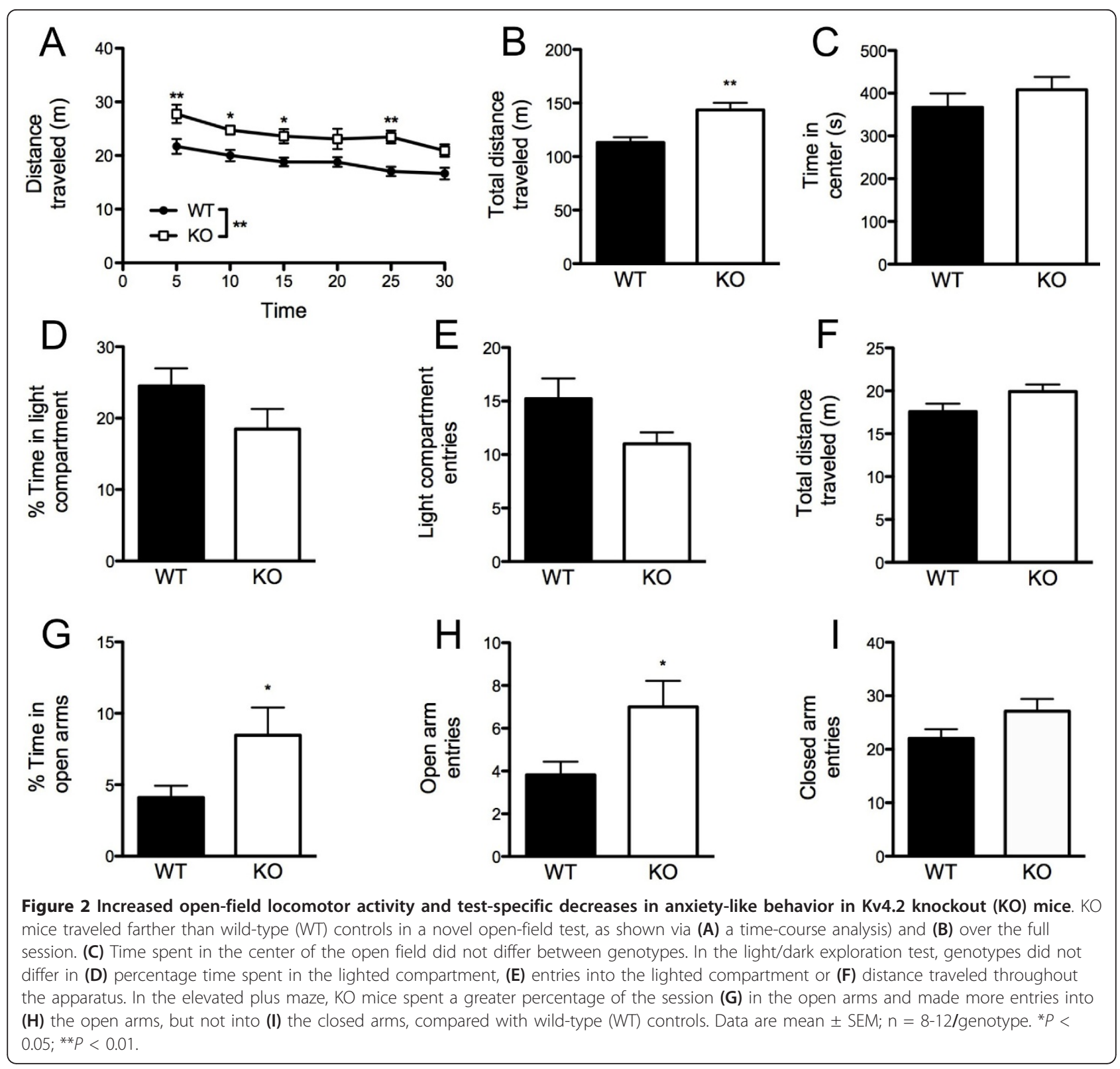

profile in the same behavioral assays after deletions of other genes involved in regulating neuronal excitability, such as components of the glutamate signaling system $[16,37,38]$. This overlap indicates that genetic loss of molecules involved in regulating neuronal excitability and synaptic plasticity produces some convergent phenotypic effects on novelty-driven behavioral reactivity. Although hyperlocomotion in a novel environment is often taken as a measure relevant to schizophrenia $[39,40]$, the Kv4.2 KO mice in the present study did not show changes in another schizophrenia-relevant measure, prepulse inhibition of startle, unlike our findings in previous studies of glutamate deletions. Therefore, without additional experiments to interrogate this issue further, it would be premature discuss the Kv4.2 KO phenotype in terms of potential relevance to that disease.

Novelty-induced hyperactivity can confound interpretation of behavior in tests for anxiety-like behavior due to non-specific increases in activity [41]. However, we found that Kv4.2 KO mice were no different from WT controls in the light/dark exploration test and showed increased open-arm exploration in the elevated plus maze, which was not associated with increased general locomotor activity in this test (as measured by the number of closed-arm entries). This suggests a test-specific anxiolytic-like phenotype in the KO mice, albeit with the caveat that the classic conflict-based assays for 

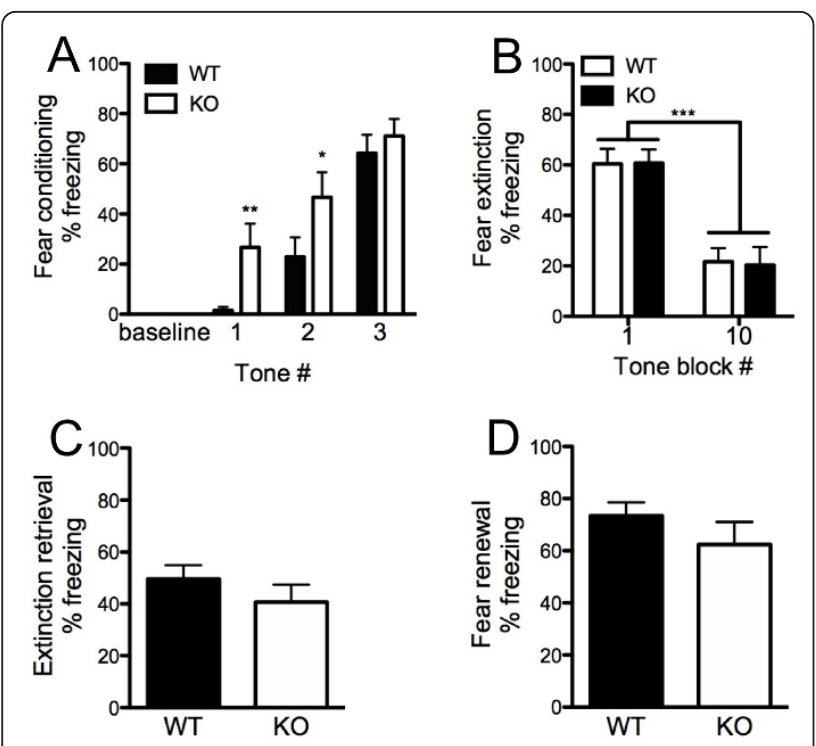

Figure 3 Increased freezing to unconditioned tones in Kv4.2 knockout (KO) mice. (A) During conditioning $\mathrm{KO}$ mice froze more than wild-type (WT) controls during the first (before conditioning) and second conditioned stimulus (CS) presentation trials. (B) Genotypes showed equivalent initial freezing and similar reductions in freezing from the first to the last five CS trial block during extinction training. Genotypes did not differ in freezing during (C) extinction retrieval or (D) fear renewal. Data are mean \pm SEM; $n=$ 8-12/genotype. ${ }^{*} P<0.05 ;{ }^{* *} P<0.01$.

anxiety-like behavior cannot unequivocally parse an anxiety-like decrease in avoidance from a novelty-driven increase in approach [41]. Notwithstanding, the test-specific nature of this phenotype is notable, and echoes earlier studies showing that the elevated plus maze can be particularly sensitive to certain gene mutations, possibly because it is inherently more stressful than ostensibly similar tests such as the light/dark exploration [42]. This would generally fit with a profile of exaggerated behavioral responses of Kv4.2 KO mice, particularly under conditions of strong environmental 'provocation.'

Consistent with this interpretation, the $\mathrm{KO}$ mice showed increased unconditioned freezing to an auditory stimulus, and an augmented corticosterone response to a single exposure to the forced-swim test. By contrast, the behavioral response (immobility) to forced swim, a very stressful and 'provocative' situation, was normal in the $\mathrm{KO}$ mice. Increases in immobility produced by repeated, brief, forced-swim exposures were also similar between KO mice and WT controls. Thus, increased behavioral reactivity in the mutants does not generalize to altered immobility during various forms of forcedswim exposure. One explanation is enhanced tonic inhibitory transmission [9] served to mitigate the penetrance of the phenotype under forced-swim conditions.
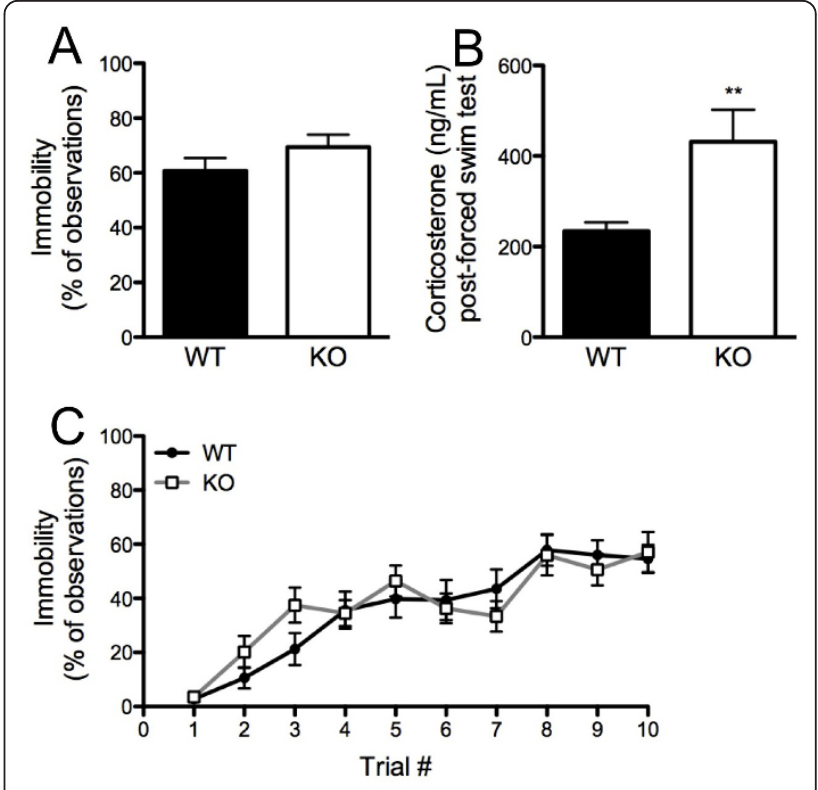

Figure 4 Exaggerated swim-induced corticosterone and normal responses to swim stressors in Kv4.2 knockout (KO) mice. (A) Genotypes did not differ in percentage immobility during a single 6-minute forced-swim exposure ( $n=9-13 /$ genotype). (B) KO mice had significantly raised serum corticosterone levels after the single forced-swim exposure ( $n=9-13 /$ genotype). (C) Genotypes had a similar increase in percentage immobility increased over $10 \times 1$-min inescapable forced swim exposures ( $n=14-18 /$ genotype). Data are mean \pm SEM. ${ }^{* *} P<0.01$.

The absence of changes in forced-swim tests in the current study differs from the previous finding by Lockridge et al. that these mice showed increased immobility in the forced-swim test [20]. Indeed, whereas the earlier report found, as we did, novel open-field hyperactivity, some other differences were seen in the earlier study, including no change in elevated plus maze anxiety-like behavior. Other than methodological variations, the principal salient factor potentially explaining these apparent discrepancies is genetic background. Kv4.2 KO mice were bred on a C57BL/6J background for the current study and a 129S6 background for the previous study. Genetic background can have a profound influence on the penetrance and expression of emotionrelated phenotypes in mutant mice, because of epistatic interactions between a mutation and modifier genes $[21,22]$. It seems likely that such interactions shaped the phenotypic profile of the Kv4.2 KO mice, and it would be interesting to explore this further by identifying the responsible modifiers.

Another important avenue for future studies will involve assessment of Kv4.2 KO mice for learning and memory. The mutants have enhanced hippocampal synaptic plasticity $[6,10]$, which is associated with altered synaptic expression of synaptic proteins that are strongly 
implicated in learning by mutant studies [23,43-45], such as the NMDA receptor GluN2A and GluN2B subunits [11-13]. In the current study we found that the KO mice displayed normal amygdala-mediated fear learning and mPFC-mediated fear extinction. Given the localization of synaptic alterations to the hippocampus, it will be valuable to supplement these findings by assessing Kv4.2 KO mice for hippocampal-dependent forms of learning.

\section{Conclusions}

In summary, we found that constitutive deletion of the A-type voltage-gated $\mathrm{K}+$ channel $\mathrm{Kv} 4.2$ produced a behavioral phenotype principally characterized by increased behavioral reactivity to exposure to some but not all novel environmental situations. This largely confirms earlier evaluation of these mice, although some discrepancies were found, possibly due to the influence of genetic background. These behavioral abnormalities extend the finding that deletion of Kv4.2 leads to exaggerated neuronal excitability in cortical and hippocampal regions involved in emotion regulation. These data shed further light on the role of Kv4.2 in regulating neural and behavioral functions.

\section{List of abbreviations}

5-HT: 5-hydroxytryptamine; ABS: acrylonitrile butadiene styrene; CS: conditioned stimulus; KO: knockout; LTD: long-term depression; LTP: longterm potentiation; mPFC: medial prefrontal cortex; NMDA: N-Methyl-Daspartate; PPI: prepulse inhibition of startle; WT: wild-type.

\section{Acknowledgements}

We thank Begum Choudhary and Heather Cameron for technical assistance. Funding was provided by the National Institute of Mental Health and the National Institute of Alcohol Abuse and Alcoholism Intramural Research Program.

\section{Author details}

'Laboratory of Behavioral and Genomic Neuroscience, National Institute on Alcohol Abuse and Alcoholism, NIH, Bethesda, MD, USA. ${ }^{2}$ Molecular Neurophysiology and Biophysics Unit, Laboratory of Cellular and Synaptic Neurophysiology, Eunice Kennedy Shriver National Institute of Child Health and Human Development, NIH, Bethesda, MD, USA.

\section{Authors' contributions}

CK carried out the behavioral testing and drafted the manuscript, DH generated the Kv4.2 mice and aided in manuscript revision, and $\mathrm{AH}$ conceived of the study and helped draft the manuscript. All authors read and approved the final manuscript.

\section{Competing interests}

The authors declare that they have no competing interests.

Received: 28 December 2011 Accepted: 2 March 2012

Published: 2 March 2012

\section{References}

1. Shah MM, Hammond RS, Hoffman DA: Dendritic ion channel trafficking and plasticity. Trends Neurosci 2010, 33(7):307-16.

2. Serodio P, Rudy B: Differential expression of Kv4 K+ channel subunits mediating subthreshold transient $\mathrm{K}+$ (A-type) currents in rat brain. $J$ Neurophysiol 1998, 79(2):1081-91.
3. Sheng $M$, et al: Subcellular segregation of two A-type $K+$ channel proteins in rat central neurons. Neuron 1992, 9(2):271-84.

4. Hoffman DA, et al: $\mathrm{K}+$ channel regulation of signal propagation in dendrites of hippocampal pyramidal neurons. Nature 1997, 387(6636):869-75

5. Kim J, Wei DS, Hoffman DA: Kv4 potassium channel subunits control action potential repolarization and frequency-dependent broadening in rat hippocampal CA1 pyramidal neurones. J Physiol 2005, 569(Pt 1):41-57.

6. Chen $\mathrm{X}$, et al: Deletion of Kv4.2 gene eliminates dendritic A-type $\mathrm{K}+$ current and enhances induction of long-term potentiation in hippocampal CA1 pyramidal neurons. J Neurosci 2006, 26(47):12143-51.

7. Nerbonne JM, et al: Electrical remodelling maintains firing properties in cortical pyramidal neurons lacking KCND2-encoded A-type K+ currents. J Physiol 2008, 586(6):1565-79.

8. Hu HJ, et al: The kv4.2 potassium channel subunit is required for pain plasticity. Neuron 2006, 50(1):89-100.

9. Andrasfalvy BK, et al: Altered synaptic and non-synaptic properties of CA1 pyramidal neurons in Kv4.2 knockout mice. J Physiol 2008, 586(16):3881-92

10. Zhao C, et al: Dendritic mechanisms controlling the threshold and timing requirement of synaptic plasticity. Hippocampus 2010.

11. Kim J, et al: Regulation of dendritic excitability by activity-dependent trafficking of the A-type $\mathrm{K}+$ channel subunit Kv4.2 in hippocampal neurons. Neuron 2007, 54(6):933-47.

12. Jung SC, Hoffman DA: Biphasic somatic A-type K channel downregulation mediates intrinsic plasticity in hippocampal CA1 pyramidal neurons. PLoS One 2009, 4(8):e6549.

13. Lei Z, et al: Downregulation of Kv4.2 channels mediated by NR2Bcontaining NMDA receptors in cultured hippocampal neurons. Neuroscience 2010, 165(2):350-62.

14. Lei Z, Deng P, Xu ZC: Regulation of Kv4.2 channels by glutamate in cultured hippocampal neurons. J Neurochem 2008, 106(1):182-92.

15. Lee $\mathrm{HY}$, et al: Bidirectional regulation of dendritic voltage-gated potassium channels by the fragile $\mathrm{x}$ mental retardation protein. Neuron 2011, 72(4):630-42

16. Boyce-Rustay JM, Holmes A: Genetic inactivation of the NMDA receptor NR2A subunit has anxiolytic- and antidepressant-like effects in mice. Neuropsychopharmacology 2006, 31(11):2405-14.

17. Kiselycznyk $C L$, et al: Genetic, pharmacological and lesion analyses reveal a selective role for corticohippocampal GLUN2B in a novel repeated swim stress paradigm. Neuroscience 2011.

18. Guo W, et al: Targeted deletion of Kv4.2 eliminates I(to, $f$ ) and results in electrical and molecular remodeling, with no evidence of ventricular hypertrophy or myocardial dysfunction. Circ Res 2005, 97(12):1342-50.

19. Barnwell LF, et al: Kv4.2 knockout mice demonstrate increased susceptibility to convulsant stimulation. Epilepsia 2009, 50(7):1741-51.

20. Lockridge A, Su J, Yuan LL: Abnormal 5-HT modulation of stress behaviors in the Kv4.2 knockout mouse. Neuroscience 2010, 170(4):1086-97.

21. Holmes A, Hariri AR: The serotonin transporter gene-linked polymorphism and negative emotionality: placing single gene effects in the context of genetic background and environment. Genes Brain Behav 2003, 2(6):332-5.

22. Crusio WE, et al: Standards for the publication of mouse mutant studies. Genes Brain Behav 2009, 8(1):1-4.

23. Brigman $\mathrm{J}$, et al: Loss of GluN2B-containing NMDA receptors in CA1 hippocampus and cortex impairs long-term depression, reduces dendritic spine density, and disrupts learning. J Neurosci 2010, 30(13):4590-600.

24. Crawley JN, Paylor R: A proposed test battery and constellations of specific behavioral paradigms to investigate the behavioral phenotypes of transgenic and knockout mice. Horm Behav 1997, 31(3):197-211.

25. Millstein RA, et al: Effects of repeated maternal separation on prepulse inhibition of startle across inbred mouse strains. Genes Brain Behav 2006, 5(4):346-54

26. Feyder M, et al: Association of mouse Dlg4 (PSD-95) gene deletion and human DLG4 gene variation with phenotypes relevant to autism spectrum disorders and Williams' syndrome. Am J Psychiatry 2010, 167(12):1508-17.

27. Hall C, Ballachey EL: A study of the rat's behavior in a field. A contribution to method in comparative psychology. University of California Publications in Psychology; 1932:6:1-12. 
28. Karlsson RM, et al: Assessment of glutamate transporter GLAST (EAAT1)deficient mice for phenotypes relevant to the negative and executive/ cognitive symptoms of schizophrenia. Neuropsychopharmacology 2009, 34(6):1578-89.

29. Mozhui K, et al: Strain differences in stress responsivity are associated with divergent amygdala gene expression and glutamate-mediated neuronal excitability. J Neurosci 2010, 30(15):5357-67.

30. Handley SL, Mithani S: Effects of alpha-adrenoceptor agonists and antagonists in a maze-exploration model of 'fear'-motivated behaviour. Naunyn Schmiedebergs Arch Pharmacol 1984, 327(1):1-5.

31. Fegley DB, et al: Increased fear- and stress-related anxiety-like behavior in mice lacking tuberoinfundibular peptide of 39 residues. Genes Brain Behav 2008, 7(8):933-42.

32. Davis AR, et al: Yohimbine impairs extinction of cocaine-conditioned place preference in an alpha2-adrenergic receptor independent process. Learn Mem 2008, 15(9):667-76.

33. Yang RJ, et al: Variation in Mouse Basolateral Amygdala Volume is Associated With Differences in Stress Reactivity and Fear Learning. Neuropsychopharmacology 2008.

34. Porsolt RD, Bertin A, Jalfre M: "Behavioural despair" in rats and mice: strain differences and the effects of imipramine. Eur J Pharmacol 1978, 51(3):291-4

35. Brigman $\mathrm{JL}$, et al: Pharmacological or genetic inactivation of the serotonin transporter improves reversal learning in mice. Cereb Cortex 2010, 20(8):1955-63.

36. Boyce-Rustay JM, Cameron HA, Holmes A: Chronic swim stress alters sensitivity to acute behavioral effects of ethanol in mice. Physiol Behav 2007, 91(1):77-86.

37. Karlsson RM, Heilig M, Holmes A: Loss of Glial Glutamate and Aspartate Transporter (Excitatory Amino Acid Transporter 1) Causes Locomotor Hyperactivity and Exaggerated Responses to Psychotomimetics: Rescue by Haloperidol and Metabotropic Glutamate 2/3 Agonist. Biol Psychiatry 2008.

38. Wiedholz LM, et al: Mice lacking the AMPA GluR1 receptor exhibit striatal hyperdopaminergia and 'schizophrenia-related' behaviors. Mol Psychiatry 2008, 13(6):631-40

39. Arguello PA, Gogos JA: Modeling madness in mice: one piece at a time. Neuron 2006, 52(1):179-96.

40. Powell CM, Miyakawa T: Schizophrenia-relevant behavioral testing in rodent models: a uniquely human disorder? Biol Psychiatry 2006, 59(12):1198-207

41. Cryan JF, Holmes A: The ascent of mouse: advances in modelling human depression and anxiety. Nat Rev Drug Discov 2005, 4(9):775-90.

42. Holmes A, et al: Galanin GAL-R1 receptor null mutant mice display increased anxiety-like behavior specific to the elevated plus-maze. Neuropsychopharmacology 2003, 28(6):1031-44.

43. Brigman $\mathrm{J}$, et al: Impaired discrimination learning in mice lacking the NMDA receptor NR2A subunit. Learn Mem 2008, 15(2):50-4.

44. Bannerman DM, et al: NMDA Receptor Subunit NR2A Is Required for Rapidly Acquired Spatial Working Memory But Not Incremental Spatial Reference Memory. J Neurosci 2008, 28(14):3623-30.

45. von Engelhardt J, et al: Contribution of hippocampal and extrahippocampal NR2B-containing NMDA receptors to performance on spatial learning tasks. Neuron 2008, 60(5):846-60.

doi:10.1186/2045-5380-2-5

Cite this article as: Kiselycznyk et al:: Effects of genetic deletion of the Kv4.2 voltage-gated potassium channel on murine anxiety-, fear- and stress-related behaviors. Biology of Mood \& Anxiety Disorders 2012 2:5.

\section{Submit your next manuscript to BioMed Central and take full advantage of:}

- Convenient online submission

- Thorough peer review

- No space constraints or color figure charges

- Immediate publication on acceptance

- Inclusion in PubMed, CAS, Scopus and Google Scholar

- Research which is freely available for redistribution 